\title{
Cavity Resonator Wireless Power Transfer System for Freely Moving Animal Experiments
}

\author{
Henry Mei, Student Member, IEEE, Kyle A. Thackston, Student Member, IEEE, Rebecca A. Bercich, \\ Student Member, IEEE, John G.R. Jefferys, and Pedro P. Irazoqui, Senior Member, IEEE
}

\begin{abstract}
Objective: The goal of this work is to create a large wireless powering arena for powering small devices implanted in freely behaving rodents. Methods: We design a cavity resonator based WPT system and utilize our previously developed optimal impedance matching (IM) methodology to achieve effective WPT performance for operating sophisticated implantable devices, made with miniature receive coils $(<8 \mathrm{~mm}$ in diameter), within a large volume (dimensions: $60.96 \mathrm{~cm} \times 60.96 \mathrm{~cm} \times 30 \mathrm{~cm}$ ). We provide unique cavity design and construction methods which maintains electromagnetic performance of the cavity while promoting its utility as a large animal husbandry environment. Additionally, we develop a biaxial receive resonator system to address device orientation insensitivity within the cavity environment. Functionality is demonstrated with chronic experiments involving rats implanted with our custom designed bioelectric recording device. Results: We demonstrate an average powering fidelity of $\mathbf{9 3 . 5 3 \%}$ over 9 recording sessions across 9 weeks, indicating nearly continuous device operation for a freely behaving rat within the large cavity resonator space. Conclusion: We have developed and demonstrated a cavity resonator based WPT system for long term experiments involving freely behaving small animals. Significance: This cavity resonator based WPT system offers an effective and simple method for wirelessly powering miniaturized devices implanted in freely moving small animals within the largest space.
\end{abstract}

Index Terms-Bioelectronics, bandpass filter, Cavity resonator, implantable devices, wireless power transfer

Copyright (c) 2016 IEEE. Personal use of this material is permitted However, permission to use this material for any other purposes must be obtained from the IEEE by sending an email to pubs-permissions@ ieee.org

Manuscript received ...; revised ...; accepted ...

This work was sponsored by the Defense Advanced Research Projects Agency (DARPA) BTO under the auspices of Dr. Doug Weber through the Space and Naval Warfare Systems Center, Grant/Contract No. N6601-14-2 4056

H. Mei, K.A. Thackston, and R.A. Bercich are with the Center for Implantable Devices, Weldon School of Biomedical Engineering, Purdue University, West Lafayette, IN 47907 USA (e-mail: hmei@purdue.edu; kthackst@purdue.edu; rbercich@purdue.edu).

J.G.R. Jefferys is with the Dept. of Pharmacology at the University of Oxford, Oxford, United Kingdom (e-mail: john.jefferys@pharm.ox.ac.uk)

P.P. Irazoqui is with the Center for Implantable Devices, Weldon School of Biomedical Engineering, and School of Electrical and Computer Engineering, Purdue University, West Lafayette, IN 47907 USA (e-mail: pip@ purdue.edu).

This paper has supplementary downloadable material available at http://ieeexplore.ieee.org, provided by the authors. This includes one PDF document and two multimedia mp4 format movie clips, which shows freely moving animal experiments within the cavity resonator. The material is $64 \mathrm{Mb}$ in size.

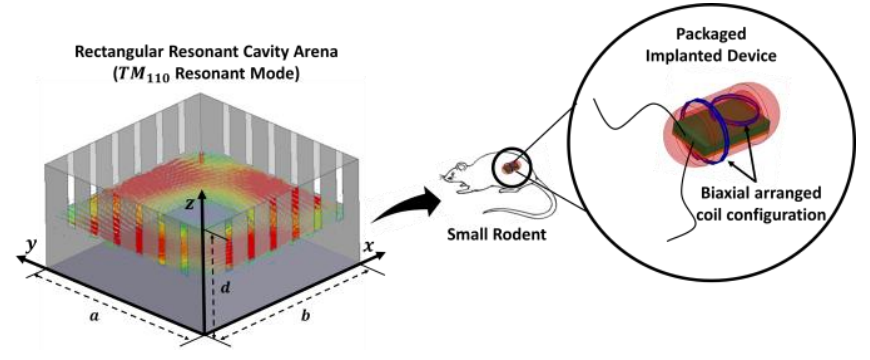

Fig. 1. Summarized illustration of cavity resonator WPT arena. The magnetic field density of the $\mathrm{TM}_{110}$ resonant mode is illustrated (Red, large; Blue, small). A small animal implanted with a custom designed device is housed within the cavity volume. A biaxial arranged power module is used to promote orientation insensitivity.

\section{INTRODUCTION}

NTEGRATION of wireless electronics with physiology has long been a goal of researchers to increase the clinical impact of animal experiments. Already, many implantable devices have been effectively integrated with wireless communication, offering bi-directional telemetry, remote control of stimulus parameters, and information processing capabilities [1-4]. In contrast, practical and efficient integration of wireless powering, remains a key challenge. Many conventional devices rely on batteries $[2,5,6]$. A major drawback of these systems, however, is the limited life-span (minutes to hours) of the battery making animal studies requiring continuous device operation, in time spans ranging from days to months, nearly impossible. This is especially true for sophisticated implantable devices with increased power consumption and functionality such as multiple recording channels with high sampling rates, wireless telemetry, optical and/or electrical stimulation, and feedback. High capacity batteries are typically too large for safe implantation in an animal [5, 7]. For these reasons, wireless power offers an attractive alternative to battery power. Toward this end, several groups have developed wireless powering arenas and enclosures for small animal studies [8-14]. These systems can be divided into those that operate using near-field induction and the recently developed mid-field method [15].

Near-field WPT systems can deliver large amounts of power, but only at short distances away from the source coils. To mitigate this shortcoming, a variety of highly engineered methods have been proposed [8, 9, 12, 13, 16-18]. Although 
these systems achieve some level of improvement, none can overcome the fundamental 2-dimensional nature of inductive coupling and the necessity for large $(>1 \mathrm{~cm})$ and often unimplantable receive coils operating in small areas around the field source.

Recently, a mid-field WPT arena for mice was proposed [19]. In that work, the researchers demonstrated the operation of a passive optogenetic stimulator implanted in mice. Due to the low power consumption (minimum of $5.6 \mathrm{~mW}$ ) and simple design (rectifier and LED), the researchers were able to utilize a $2 \mathrm{~mm}$ diameter receive coil for power capture, the smallest of its kind for an optogenetic device. Nevertheless, that WPT system does not resolve the persisting need for a miniaturization strategy which maintains an ability to continuously wirelessly power more sophisticated and power-hungry devices. Moreover, the energy coupling was confined to an enclosure for mice which employed a working height of $3 \mathrm{~cm}$ and a circular surface of $21 \mathrm{~cm}$ in diameter. That system is unsuitable as a non-restrictive environment for rats.

In this work, we introduce a new WPT arena, built of a uniquely designed cavity resonator, for longitudinal studies involving freely behaving small animals. Our system achieves effective wireless powering to miniature sized receive coils for powering sophisticated implantable devices in large volumes. Fig. 1 provides a summarized illustration of our WPT approach. Our ability to operate with small receive coils, within the large volume $\left(108,050 \mathrm{~cm}^{3}\right)$, is due to two critical points: 1$)$ we utilize our previously developed equivalent bandpass filter (BPF) circuit model and optimal impedance matching (IM) methodology to predict and attain maximum achievable PTE's [20] and 2) the unloaded Q-factor $\left(\mathrm{Q}_{0}\right)$ of the cavity resonator is naturally high, which maximizes the system's attainable PTE [21].

We achieve suitable orientation insensitivity of the WPT receiver by implementing a uniquely designed biaxial receive resonator system in which each axis can be tuned individually using our optimal IM methodology. We excite the cavity resonator at its dominant mode, termed the $\mathrm{TM}_{110}$ mode, where TM is transverse magnetic and sub-scripts 1,1 , and 0 represents the standing wave pattern. Previously developed cavity resonator WPT systems have utilized the excitation of higher order modes to achieve orientation insensitivity [22]. However, excitation of higher order resonant modes requires excitation at multiple frequencies. As an implanted device is tuned to a single resonant frequency, adaptability to higher order excitation modes would require changing on-board impedance matching, which is impractical for an implanted device. Thus, multi-mode excitation is not utilized in this application.

To demonstrate the functionality of our cavity resonator WPT method, we conduct chronic in-vivo experiments involving freely behaving rats implanted with a custom designed multichannel bioelectric recording device measuring ECG signals.

\section{CAVity Resonator Design AND CONSTRUCtion}

Fig. 2 shows the constructed custom designed rectangular cavity resonator and Table I provides a summary of relevant dimensions. A cavity resonator is any hollow closed conductor

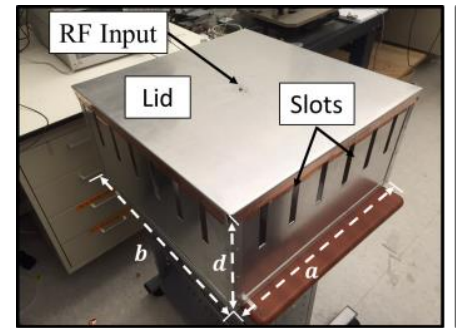

(a)

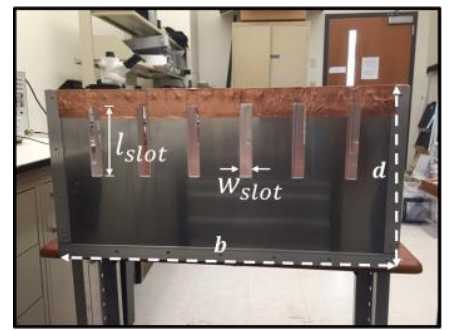

(c)

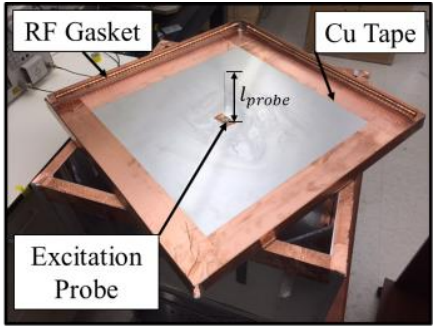

(b)

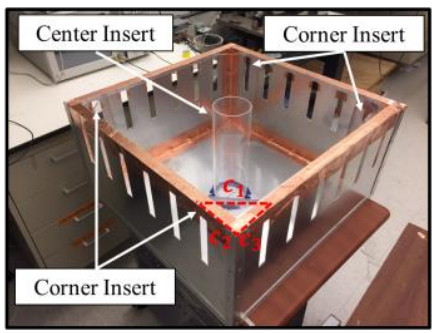

(d)
Fig. 2. Photograph of constructed cavity resonator. (a) Full perspective view with lid attached. (b) Underside of lid unattached to cavity. The excitation probe with length, $l_{\text {probe }}$, is located at the center of the lid. The lip of the lid is lined with RF gasket. Copper tape is utilized to reinforce electrical connections. (c) Side view of cavity resonator with detailed view of the cut out slots. (d) Full perspective view without lid. Interior "blind-spot" barriers are shown.

TABLE I

ALUMINUM CAVITY RESONATOR DIMENSIONS

\begin{tabular}{|c|c|}
\hline Dimension & Value \\
\hline$a$ & $60.96 \mathrm{~cm}$ \\
\hline$b$ & $60.96 \mathrm{~cm}$ \\
\hline$d$ & $30 \mathrm{~cm}$ \\
\hline$l_{\text {slot }}$ & $15 \mathrm{~cm}$ \\
\hline$w_{\text {slot }}$ & $2.1 \mathrm{~cm}$ \\
\hline Center Barrier Radius & $5 \mathrm{~cm}$ \\
\hline Corner Barrier $\left(c_{1}, c_{2}, c_{3}\right)$ & $5 \mathrm{~cm}, 3.54 \mathrm{~cm}, 3.54 \mathrm{~cm}$ \\
\hline
\end{tabular}

that can give rise to modes of electromagnetic (EM) standing waves reflecting back and forth between the cavity walls. We design a novel cavity resonator that maintains EM properties while incorporating design considerations that allow it to be used for long-term small animal housing. These include large physical dimensions, viewing and ventilation slots, and an easily removable lid for animal transport and structural integrity. This system was approved for use by the Purdue Animal Care and Use Committee (PACUC) at Purdue University and is compliant with the UK Animals (Scientific Procedures) Act of 1986 and approved for use at the University of Oxford.

\section{A. Material Selection}

A cavity can be constructed out of any conductive material. High conductivity material, however, is critical for maximizing cavity unloaded quality factor, $Q_{0}$; the ratio of stored energy over energy losses per cycle [23]. In cavity resonator structures, losses are primarily due to conductor loss and the presence of an electrically lossy object within the cavity. Importantly, high $Q_{0}$ is essential to maximizing the system's highest attainable PTE [22]. To enable adoption by the scientific community, we construct the cavity using aluminum alloy 1100 (99\% aluminum content). We also construct a cavity of equivalent 


\section{$>$ REPLACE THIS LINE WITH YOUR PAPER IDENTIFICATION NUMBER (DOUBLE-CLICK HERE TO EDIT) <}

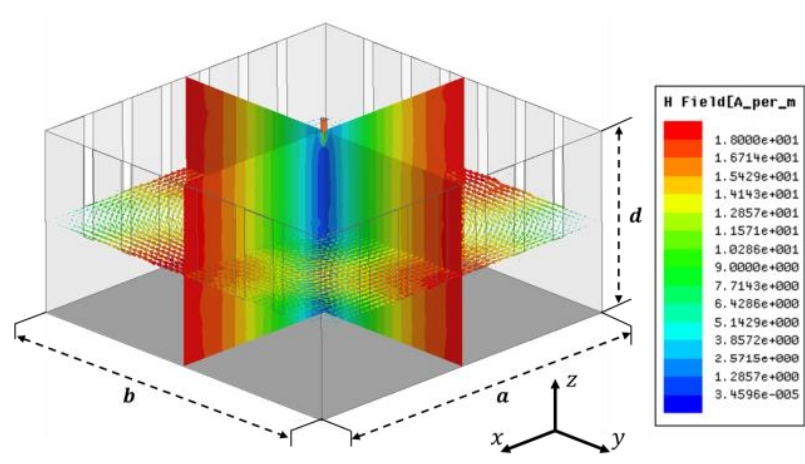

Fig. 3. Simulated $\vec{H}$-field distribution in the $\mathrm{TM}_{110}$ excitation mode.

dimensions using super-conductive 101 copper (99.99\% copper content) to compare tradeoffs in cost versus WPT performance [Suppl. Fig. 1]. Due to the accessibility of aluminum, all in-vivo experiments were conducted using the aluminum cavity.

\section{B. Cavity Dimensions and Resonant Frequency}

The cavity resonator structure acts as the primary housing enclosure and was designed with dimensions of $a=60.96 \mathrm{~cm}$, $b=60.96 \mathrm{~cm}, d=30$. The large dimensions facilitate normal animal movement. Additionally, bedding, absorbing mats, and food can be placed within the enclosure to enhance animal welfare.

The resonant frequency, $f_{110}$, of a rectangular cavity, where the height is less than the length and width, excited in the $\mathrm{TM}_{110}$ mode, is calculated by

$$
f_{110}=\frac{c}{2 \pi \sqrt{\mu_{r} \varepsilon_{r}}} \sqrt{\left(\frac{\pi}{a}\right)^{2}+\left(\frac{\pi}{b}\right)^{2}}
$$

where $c, \mu_{r}$, and $\varepsilon_{r}$ are the speed of light, relative magnetic permeability and relative dielectric permittivity of a material within the cavity, respectively [23]. Using (1), our cavity has a predicted $f_{110}$ of $347.99 \mathrm{MHz}$-well below the $\mathrm{GHz}$ frequencies at which wireless powering becomes ineffective at powering most implantable wireless sensors [24].

\section{C. $\vec{H}$-Field Distribution in the TM110 Excitation Mode}

Fig. 3 shows the simulated magnetic field $(\vec{H}$-field $)$ distribution of the cavity excited in the transverse magnetic (TM) $\mathrm{TM}_{110}$ mode. All EM simulations were conducted using ANSYS High Frequency Structural Simulator $\left(\mathrm{HFSS}^{\circledR}\right)$. The $\vec{H}$-field is rotational about the center of the cavity, increases in magnitude radially, and is not a function of height. We utilize a biaxial receive resonator which has an ability to capture the $\vec{H}$ field components in two axes, enabling adequate orientation insensitivity for a device implanted in a freely-behaving animal. As shown in Fig. 3, five distinct locations (center and four corners) exhibit low-field strength. Placing EM transparent barriers in these areas restricts the freely behaving animal from entering WPT "blind spots" [Fig. 2(d)].

\section{Cavity Slots and Animal Viewing}

Cutting out six slots on the top of each wall of the cavity

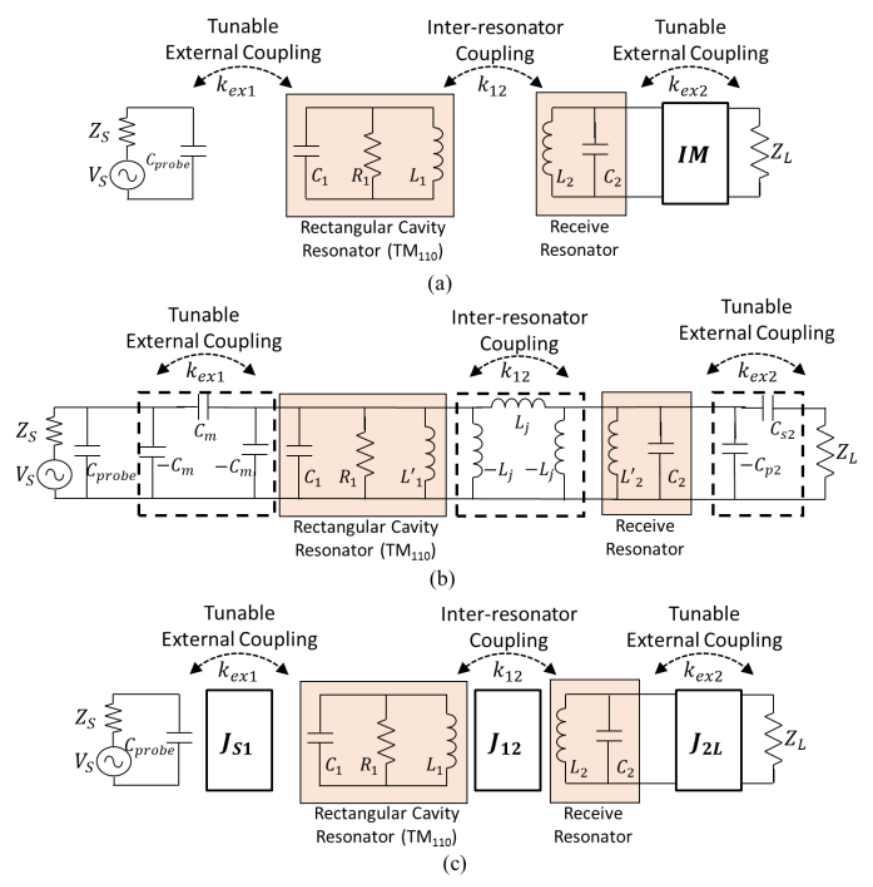

Fig. 4. (a) BPF circuit model of cylindrical cavity WPT system. (b) Equivalent circuit with equivalent electric and magnetic coupling networks. (c) Representation of external coupling networks as admittance inverters (Jinverters).

enables ease of animal viewing, permits air flow within the cavity to maintain temperature and humidity requirements, and water access (water spout). Additionally, by designing the width of the slot, $w_{\text {slot }}$, to be $2.1 \mathrm{~cm}$, which is $<<\lambda_{110}$, where $\lambda_{110}$ is the wavelength at the $\mathrm{TM}_{110}$ frequency $(\approx 86.5 \mathrm{~cm})$, we minimize radiative losses.

\section{E. Removable Lid Design and Excitation Probe}

Incorporating a removable lid enables animal placement and housing maintenance (cleaning, water and food refilling, bedding replacement, and sanitization). Copper beryllium metal gaskets (Laird Technologies EMI, manufacturing part no. 0097064002) at the lips of the lid ensure robust electrical connection between the removable lid and the cavity. A coaxial probe with a length, $l_{\text {probe }}$, electrically couples radiofrequency (RF) energy into the cavity and is connected to the lid via a subminiature type-A (SMA) connector where RF ground is connected directly to the cavity [Fig. 1(a)]. A cylindrical barrier (Diameter $=10 \mathrm{~cm}$ ) is placed in the center of the cavity to prevent interaction between the animal and excitation probe. As will be described in Sections III and IV, the length of the probe is critical for attaining maximum achievable PTE.

\section{CAVITY RESONATOR WPT CiRCUIT MODEL AND OPTIMIZATION}

Our unique circuit model (Fig. 4) and microwave network analysis methodology to optimize impedance matching (IM) for greatest PTE is presented and is supported by measured data.

\section{A. Equivalent System Circuit Model}

Fig. 4(a) shows the equivalent circuit model of a resonant cavity, excited in the $\mathrm{TM}_{110}$ resonant mode, containing a resonant receiver (coil inductor and capacitor) connected to a 


\section{$>$ REPLACE THIS LINE WITH YOUR PAPER IDENTIFICATION NUMBER (DOUBLE-CLICK HERE TO EDIT) <}

load. In this first order mode, we model the cavity as a parallel RLC circuit and the excitation probe as a lumped capacitor, $C_{\text {probe }}$, that electrically couples energy to excite the cavity. We design the receive resonator to resonate at the same frequency $\left(f_{110}\right)$ as the $\mathrm{TM}_{110}$ mode. Based on the parallel resonator model for the cavity and receiver resonator system, $f_{110}$ has the relationship given by

$$
f_{110}=\frac{1}{2 \pi \sqrt{L_{n} C_{n}}} \text { for } \mathrm{n}=1,2
$$

where $L_{n}$ and $C_{n}$ represent the LC pair of each resonator at $f_{110}$.

\section{B. Lumped Element J-inverter Equivalence}

The external and inter-resonator couplings in Fig. 4(a) can be replaced with their respective equivalent networks (modeled as admittance inverters (J-inverter) exhibiting real characteristic admittances of $J_{S 1}, J_{12}$, and $J_{2 L}$ ) [Fig. 4(b)]. This results in the final BPF circuit form of the cavity resonator WPT system shown in Fig. 4(c). We achieve optimal IM by configuring the external coupling lumped element networks to exhibit optimally calculated values of characteristic admittances $J_{S 1}$ and $J_{2 L}$.

The capacitive coupling between the excitation probe and the cavity resonator is replaced by the equivalent pi network, built of the mutual capacitance, $C_{m}$ [25]. This network models as an admittance inverter with characteristic admittance $J_{S 1}$ found in [26] to be

$$
J_{S 1}=\omega_{110} C_{m}=2 \pi f_{110} C_{m} .
$$

As will be shown in Section IV, $C_{m}$ varies as a function of the excitation probe length, $l_{\text {probe }}$. Therefore, the value of $J_{S 1}$ can be configured to an optimally calculated value by varying $l_{\text {probe }}$.

The inductive coupling between resonators can be replaced by its equivalent pi network with inductance of $L_{j}$ and transformations of $L_{1}$ and $L_{2}$ into $L_{1}{ }^{\prime}$ and $L_{2}{ }^{\prime}$, respectively. These relationships are

$$
\begin{gathered}
L_{j}=\frac{\left(1-k_{12}^{2}\right) \sqrt{L_{1} L_{2}}}{k_{12}} \\
L_{n}^{\prime}=L_{n}\left(1-k_{12}^{2}\right) \text { for } n=1,2 \\
k_{12}=\frac{L_{m}}{\sqrt{L_{1} L_{2}}}
\end{gathered}
$$

where $L_{m}$ is the mutual inductance. The characteristic admittance for the pi network with inductance $L_{j}$ is given by [26]

$$
J_{12}=\frac{1}{\omega_{110} L_{j}}=\frac{k_{12}}{\left(1-{k_{12}}^{2}\right) \omega_{110} \sqrt{L_{1} L_{2}}}
$$

In (3)-(6), the $\left(1-k_{12}^{2}\right)$ term will reduce to $\sim 1$, given that $k_{12} \ll 0.1$ due to the loose coupling between the cavity and receive resonator.

The IM network between the receive resonator and the load is implemented with a half pi J-inverter network consisting of a shunt capacitance, $-C_{p 2}$, and series capacitance, $C_{s 2}$. The shunt capacitance is negative in value to virtualize its function as a $\mathrm{J}$ inverter. It is absorbed by the parallel capacitor of the receive $L C$ resonator. The input admittance, $Y_{i n}$, looking into the $\mathrm{J}$ inverter located between the receive resonator and $Z_{L}$ is given by

$$
Y_{i n}=\frac{J_{2 L}^{2}}{Y_{L}}
$$

where $Y_{L}=1 / Z_{L}$. We set the source and load impedances, $Z_{s}$ and $Z_{L}$ respectively, to $50 \Omega$, to enable convenient measurement with standard $50 \Omega$ equipment. From (8), $Y_{\text {in }}$ can be expanded as

$$
Y_{i n}=j \omega_{110}\left(-C_{p 2}\right)+\left(\frac{1}{j \omega_{110} C_{s 2}}+\frac{1}{Y_{L}}\right)^{-1}
$$

where $j=\sqrt{-1}$. We substitute (9) into (8) and set real and imaginary parts equal. This produces a set of simultaneous equations where the capacitances, $-C_{p 2}$ and $C_{s 2}$, as related to $J_{2 L}$, are solved as

$$
\begin{gathered}
-C_{p 2}=\frac{J_{2 L} \sqrt{\left(1-J_{2 L}^{2} Z_{L}^{2}\right)}}{\omega_{110}} \\
C_{s 2}=\frac{J_{2 L}}{\omega_{110} \sqrt{\left(1-J_{2 L}^{2} Z_{L}^{2}\right)}}
\end{gathered}
$$

Using (10) and (11), we calculate the values of $-C_{p 2}$ and $C_{s 2}$, of which are integrated onto the receive system, to configure the IM network to exhibit an optimally calculated value of $J_{2 L}$.

\section{Microwave Network Analysis}

The cavity resonator BPF circuit model in Fig. 4(c) is a cascade of lumped element networks and J-inverters, lending itself to $\mathrm{ABCD}$ matrix representation. The $\mathrm{ABCD}$ matrix of any shunt element or resonator (not including source and load impedances) is

$$
\boldsymbol{A}_{\boldsymbol{i}}=\left[\begin{array}{cc}
1 & 0 \\
Y_{i} & 1
\end{array}\right]
$$

where $Y_{i}$ is the admittance of element $i$. The ABCD matrix of the admittance inverters are found in [23] to be:

$$
\boldsymbol{A}_{\boldsymbol{J}}=\left[\begin{array}{cc}
0 & -\frac{1}{j J_{i}} \\
j J_{i} & 0
\end{array}\right]
$$




\section{$>$ REPLACE THIS LINE WITH YOUR PAPER IDENTIFICATION NUMBER (DOUBLE-CLICK HERE TO EDIT) <}

The ABCD matrix for the entire network is then

$$
\left[\begin{array}{ll}
A_{t o t} & B_{t o t} \\
C_{t o t} & D_{t o t}
\end{array}\right]=\boldsymbol{A}_{\boldsymbol{p}} \cdot \boldsymbol{A}_{\boldsymbol{J S} \mathbf{1}} \cdot \boldsymbol{A}_{\boldsymbol{r 1}} \cdot \boldsymbol{A}_{\boldsymbol{J 1 2}} \cdot \boldsymbol{A}_{\boldsymbol{r} \mathbf{2}} \cdot \boldsymbol{A}_{\boldsymbol{J} \mathbf{L}}
$$

and the admittances of the probe, $Y_{p}$, cavity resonator, $Y_{r 1}$, and receive resonator, $Y_{r 2}$, are:

$$
\begin{gathered}
Y_{p}=j \omega C_{\text {probe }} \\
Y_{r 1}=j \omega C_{1}+\frac{1}{R_{1}}+\frac{1}{j \omega L_{1}} \\
Y_{r 2}=\frac{1}{\left(j \omega L_{2}\right)+R_{p 2}}+j \omega C_{2}
\end{gathered}
$$

where $R_{p 2}$ in (17) is included to account for the frequency dependent parasitic series resistance of the receive coil. The resistances, $R_{1}$ and $R_{p 2}$, are related to $Q_{0}$ for each resonator by:

$$
\begin{gathered}
R_{1}=Q_{01} \omega_{110} L_{1} \\
R_{p 2}=\frac{\omega_{110} L_{2}}{Q_{02}}
\end{gathered}
$$

From (7) and (15) - (19), the entire ABCD matrix can be expanded in terms of measurable and controllable design parameters i.e. $C_{\text {probe }}, C_{m}, C_{1}, C_{2}, L_{1}, L_{2}, Q_{01}, Q_{02}, k_{12}$, and unknown characteristic admittances $J_{S 1}$ and $J_{2 L}$. Using standard conversion [23], the power transmission coefficient, $S_{21}$, is given by

$$
S_{21}=\frac{2}{A_{t o t}+\left(B_{t o t} / Z_{0}\right)+C_{t o t} Z_{0}+D_{t o t}}
$$

where $Z_{0}=Z_{s}=Z_{L}=50 \Omega$. The PTE is related to $S_{21}$ by

$$
P T E=\left|S_{21}\right|^{2} \times 100 .
$$

Note that equation (21) does not include the power loss due to source impedance.

\section{Derivation of $J_{S 1 \text { opt }}$ and $J_{2 \text { Lopt }}$}

In (20), $\left|S_{21}\right|^{2}$ (PTE) is a function of optimizable parameters $J_{S 1}$ and $J_{2 L}$. As shown in Fig. 5, for a given set of initial system parameters, there exists a unique value of $J_{S 1}$ and $J_{2 L}$, renamed $J_{S 1 \text { opt }}$ and $J_{2 \text { Lopt }}$ respectively, at which $\left|S_{21}\right|^{2}$ will be a global maximum. Setting the partial derivative of (20) with respect to $J_{S 1}$ and $J_{2 L}$ to zero yields two equations that we solve simultaneously for $J_{S 1 \text { opt }}$ and $J_{2 \text { Lopt }}$. The magnitude of the resulting functions are derived as

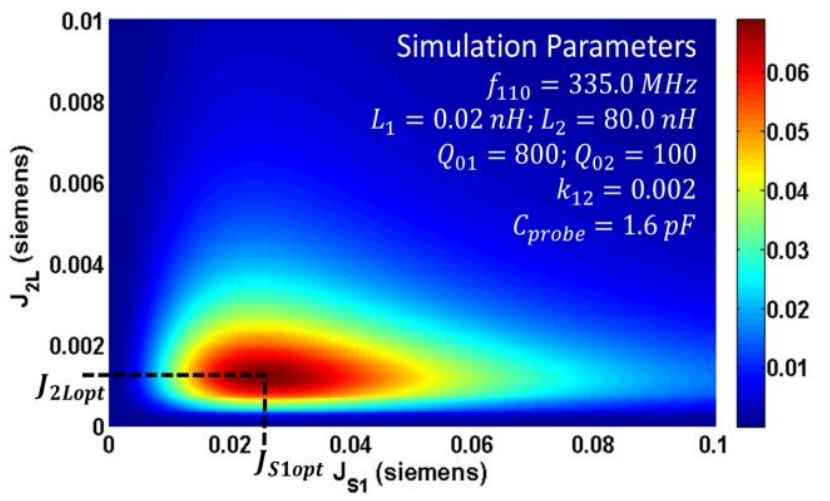

Fig. 5. Plot of $\left|S_{21}\right|^{2}$ as a function of $J_{S 1}$ and $J_{2 L}$. The location of peak $\left|S_{21}\right|^{2}$ occurs at $J_{\text {S1opt }}$ and $J_{2 \text { Lopt }}$. Simulation design parameters are given.

$$
\begin{gathered}
J_{\text {S1 opt }}=\frac{\left[\left(1+Y_{p} Z_{0}\right) \sqrt{Y_{r 1} Y_{r 2}\left(J_{12}^{2}+Y_{r 1} Y_{r 2}\right)}\right]^{1 / 2}}{\sqrt{Y_{r 2} Z_{0}}} \\
J_{2 \text { Lopt }}=\frac{\left[Y_{r 2}\left(J_{12}^{2}+Y_{r 1} Y_{r 2}\right)\right]^{1 / 4}}{Y_{r 1}^{1 / 4} \sqrt{Z_{0}}}
\end{gathered}
$$

From (22) and (23), $J_{S 1 \text { opt }}$ and $J_{2 L o p t}$ are calculated from system design parameters i.e. source, load, and resonator admittances and a specified $k_{12}$ value [represented by $J_{12}$ through (7)]. We substitute (22) into (3) and (23) into (10) and (11) to determine the capacitance values, $C_{m},-C_{p 2}$, and $C_{s 2}$, that will result in the optimal IM condition for the set of initial design parameters. Additionally, we substitute (22) and (23), along with the set of known design parameters, into (20) to predict the optimized $S_{21}$ response.

\section{DESIGN EXAMPLE AND EXPERIMENTAL VALIDATION}

This section contains a design example and measurements to validate our model. All S-parameter measurements come from standard $50 \Omega$ one-port and two-port vector network analyzer (VNA) (Agilent E5072A) measurements. All circuit simulations were performed with Agilent Advanced Design System $\left(\operatorname{ADS}^{\odot}\right)$.

\section{A. Measurement of Resonators and Design Parameters}

The experimental procedure for maximizing the performance of the cavity resonator WPT system begins by determining the cavity and receive resonator parameters, $f_{110}, C_{n}, L_{n}$, and $Q_{0 n}$. This is followed by the determination of $C_{\text {probe }}$ and $C_{m}$, as related to $l_{\text {probe }}$, and approximate $k_{12}$ values between the cavity and a receive coil.

The cavity resonator parameters $\left(f_{110}, Q_{01}, C_{1}, L_{1}\right)$ can be obtained by utilizing the one-port reflection measurement method described in [27]. This provides $f_{110}$ and $Q_{01}$ of the cavity from which we calculate the equivalent lumped parameters, $C_{1}$ and $L_{1}$, using standard microwave resonator theory.

For the receive resonator, a $7 \mathrm{~mm}$ diameter, two tightly wound turn receive coil made of 22 American wire gauge 


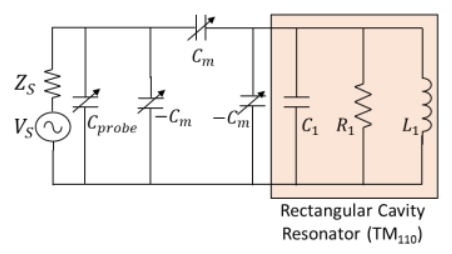

(a)

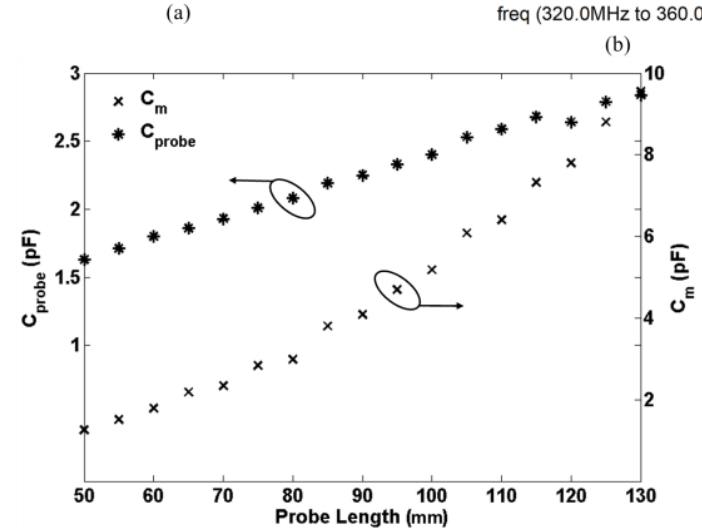

(c)

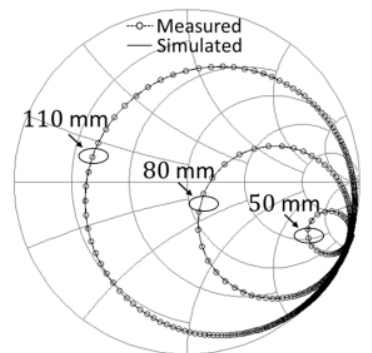

freq $(320.0 \mathrm{MHz}$ to $360.0 \mathrm{MHz})$
Fig. 6. Summary for the determination of $C_{\text {probe }}$ and $C_{m}$. (a) Circuit model used in simulation. $C_{\text {probe }}$ and $C_{m}$ are dynamically tuned in simulation until the simulated $S_{11}$ response matches to the measured response. (b) example of a circuit simulation matching to the measure response at probe lengths of 110 $\mathrm{mm}, 80 \mathrm{~mm}$, and $50 \mathrm{~mm}$. (c) plot of $C_{\text {probe }}$ and $C_{m}$ as a function of probe length.

(AWG) $\mathrm{Cu}$ magnet wire couples energy within the cavity. An $S_{11}$ recording of this coil outside of the chamber directly provides $L_{2}$ and $Q_{02}$. The resonant capacitor, $C_{2}$, comes from (2), where $L_{2}$ and $f_{110}$ are known.

We determine $C_{\text {probe }}$ and $C_{m}$ by curve fitting circuit simulations of the model shown in Fig. 6(a) to measured Sparameters, where $R_{1}, C_{1}$, and $L_{1}$ are known (from measurement) and $Z_{s}=50 \Omega$. Fig. $6(\mathrm{~b})$ presents example matches between circuit simulation and measured $S_{11}$ responses at probe lengths of $110 \mathrm{~mm}\left(C_{\text {probe }}=2.59 \mathrm{pF}, C_{m}=6.41 \mathrm{pF}\right)$ $80 \mathrm{~mm}\left(C_{\text {probe }}=2.19 \mathrm{pF}, C_{m}=3.80 \mathrm{pF}\right)$, and $50 \mathrm{~mm}$ $\left(C_{\text {probe }}=1.63 \mathrm{pF}, C_{m}=1.26 \mathrm{pF}\right)$. Fig. $6(\mathrm{c})$ presents $C_{\text {probe }}$ and $C_{m}$ versus $l_{\text {probe }}$ at values ranging from $50 \mathrm{~mm}-130 \mathrm{~mm}$ in $5 \mathrm{~mm}$ increments.

The final design parameter required for optimization is $k_{12}$ : the magnetic coupling coefficient between the coupled resonators. We estimate $k_{12}$ using the EM field solver, HFSS ${ }^{\circledR}$. Due to the relative size difference between the miniature receive coil and large cavity volume, $k_{12}$ exhibits a maximum value of 0.003 near the walls of the cavity and $k_{12}$ of near 0 directly at the center.

For the remainder of this work, we use $k_{12}=0.002$ as the coupling point where optimal IM is desired. This provides a buffer of maximized PTE between the higher $k_{12}$ values near the cavity walls and the lower $k_{12}$ values near the cavity center. Table II provides a summary of the initial system design parameters from which we calculate $J_{S 10 p t}$ and $J_{2 \text { Lopt }}$.

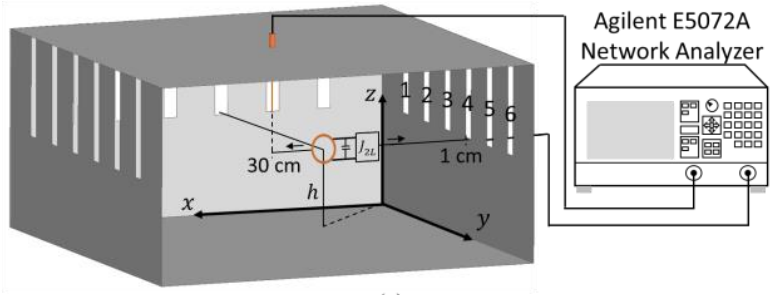

(a)

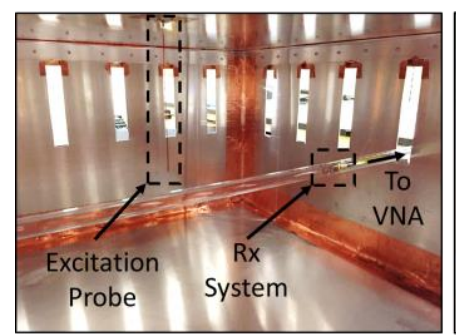

(b)

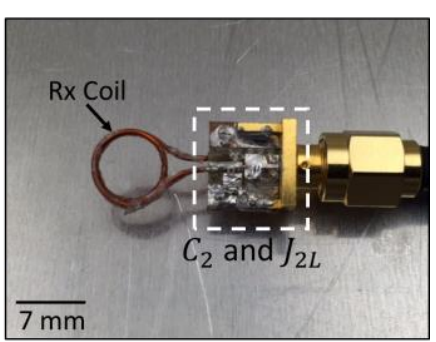

(c)
Fig. 7. Experimental set-up of cavity characterization. (a) Diagram illustration of measurement set-up and labeled positions. The receive resonator is placed through slot 4 and moved in the $\mathrm{x}$-direction. (b) Closed lid view highlighting excitation probe and receive system within plastic guide tube. (c) $7 \mathrm{~mm}$ diameter receive coil connected to the tuning PCB for measurement.

TABLE II

MEASURED RESONATOR AND INITIAL DESIGN PARAMETERS

\begin{tabular}{|c|c|c|}
\hline Parameters & $\begin{array}{c}\text { Aluminum Cavity } \\
\text { Resonator }\end{array}$ & Receive Resonator \\
\hline$f_{110}$ & $346.6 \mathrm{MHz}$ & $346.6 \mathrm{MHz}$ \\
\hline$L_{n}$ & $0.19455 \mathrm{nH}$ & $68.0 \mathrm{nH}$ \\
\hline$Q_{0 n}$ & 1027 & 67.48 \\
\hline$C_{\mathrm{n}}$ & $1083.8 \mathrm{pF}$ & $3.10 \mathrm{pF}$ \\
\hline$l_{\text {probe }}$ & $84 \mathrm{~mm}$ & $\mathrm{n} / \mathrm{a}$ \\
\hline$C_{m}$ & $3.55 \mathrm{pF}$ & $\mathrm{n} / \mathrm{a}$ \\
\hline$C_{\text {probe }}$ & $2.16 \mathrm{pF}$ & $\mathrm{n} / \mathrm{a}$ \\
\hline$k_{12}$ & \multicolumn{2}{|c|}{0.002} \\
\hline
\end{tabular}

$\mathrm{n} / \mathrm{a}=$ not applicable

TABLE III

SUMMARY OF $J_{S 1 o p t}, J_{2 L o p t}$ AND CORRESPONDING CAPACITANCE VALUES AT $\omega=\omega_{110}$ AND $k_{12}=0.002$

\begin{tabular}{|c|c|c|c|}
\hline \multirow{3}{*}{ Optimal } & $\begin{array}{c}J_{S 10 p t}= \\
0.0073\end{array}$ & \multicolumn{2}{|c|}{$J_{2 \text { Lopt }}=0.0015$} \\
\cline { 2 - 4 } & $C_{m}(\mathrm{pF})$ & $\begin{array}{c}-C_{p 2}+C_{2} \\
(\mathrm{pF})\end{array}$ & $C_{s 2}(\mathrm{pF})$ \\
\hline Calculated & 3.35 & 2.41 & 0.69 \\
\hline Implemented & $3.55^{*}$ & 2.7 & 0.7 \\
\hline
\end{tabular}

*Corresponds to $l_{\text {probe }}$ of $84 \mathrm{~mm}$

\section{B. Measured, Simulated, and Theoretically Predicted Optimal PTE Response}

Table III summarizes the calculated $J_{\text {S1opt }}, J_{\text {LLopt }}$ and corresponding capacitance values needed for achieving the optimal IM condition given the initial system design parameters summarized in Table II. We use larger $-C_{p 2}+C_{2}$ values in actual implementation to account for the high frequency variation associated with surface mount 0201 capacitors (Johansson Technology, Inc.) implemented on the printed circuit board (PCB). Setting $l_{\text {probe }}$ to $84 \mathrm{~mm}$ makes $C_{m}$ match closely with the optimally calculated value.

In Fig. 7, we show the 2-port VNA experimental set-up used for measuring $S_{21}$ [PTE through (21)] as a function of radial 


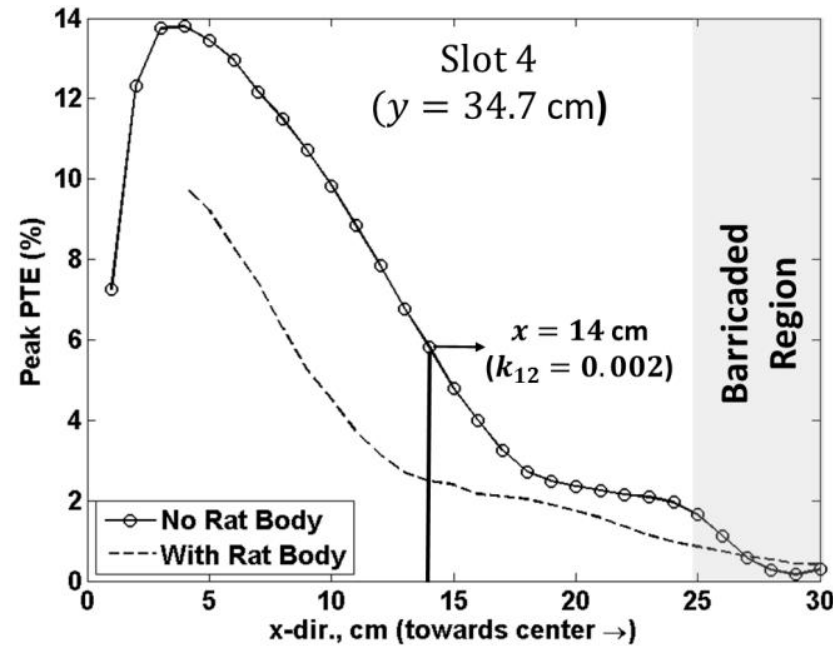

(a)

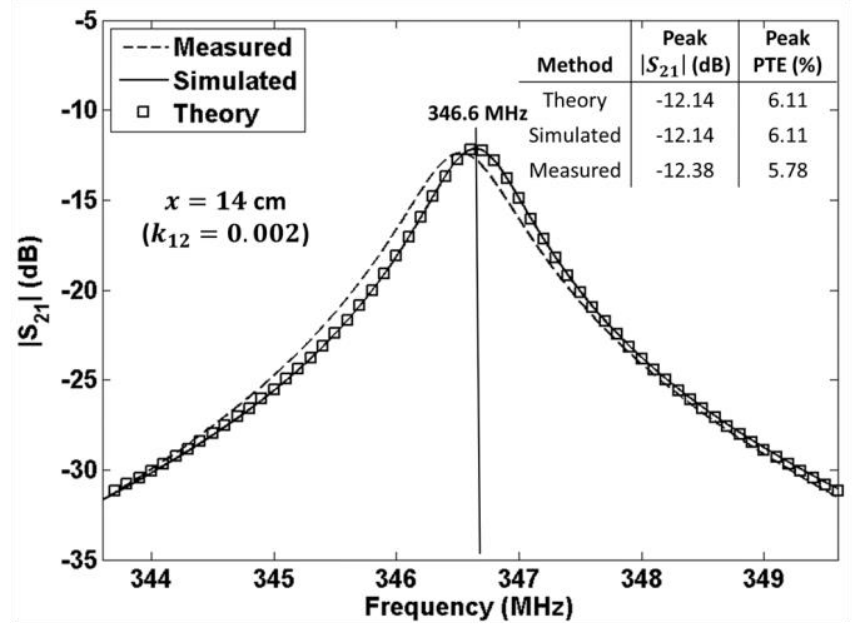

(b)

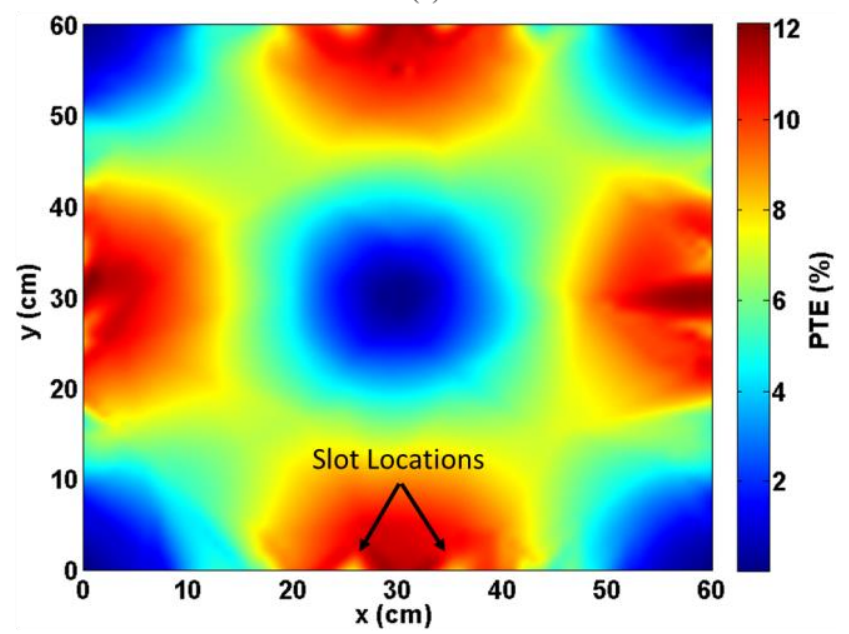

(c)

Fig. 8. Summary of measured PTE response within the cavity. Coordinates and location of measurement are in reference to Fig. 7(a). (a) Peak PTE (with and without a rat body) as a function of $\mathrm{x}$-direction position. The barricaded region, where an animal cannot enter, is highlighted. (b) Measured, circuit simulated, and theoretically predicted frequency response at $k_{12}=0.002$. (c) Measured PTE in the $x-y$ plane at $z=15 \mathrm{~cm}$. position. Moving the receive system [Fig. 7(c)] through a plastic guide tube allows us to map PTE as a function of location. As shown in Fig. 8(a), we achieve a peak PTE of $14.32 \%$ when the receiver is placed at $x=4 \mathrm{~cm}, y=34.7 \mathrm{~cm}$ (slot 4) without the receive system implanted in a rat body located within the cavity. Note, Slot 4 is the located in the middle of the cavity and was chosen to maximize coupling to the receive resonator. The peak PTE decreases as the receiver approaches the center of the cavity, verifying the $\mathrm{TM}_{110} \vec{H}$-field behavior of the cavity [see Fig. 3]. Radiative losses from the slots cause the initial low PTE when the coil enters the cavity. Fig. 8(b), illustrates the measured, circuit simulated, and theoretically predicted frequency response of the optimal IM cavity resonator WPT system at the designed point of $k_{12}=$ 0.002. A 2-dimensional plot of peak obtainable PTE, without a rat body, at $z=15 \mathrm{~cm}$ is provided in Fig. 8(c).

\section{Copper Cavity Performance}

Due to its superior conductivity, the copper cavity achieves a measured $Q_{0}$ of 12,157, which is $\sim 12$ times the measured $Q_{0}$ of the aluminum cavity. Suppl. Fig. 1(e) shows the resulting WPT performance capability of the copper cavity using smaller $5 \mathrm{~mm}$ diameter and $2 \mathrm{~mm}$ diameter coils. Using the same experimental procedure described previously, we achieved a maximum peak PTE of $51 \%$ and $7.7 \%$ to the $5 \mathrm{~mm}$ diameter [Suppl. Fig. 1(c)] and $2 \mathrm{~mm}$ diameter [Suppl. Fig. 1(d)] receive coils, respectively. This performance enhancement may justify the increased cost for researchers with greater design constraints.

\section{Accounting for Presence of the Animal in WPT}

Also shown in Fig. 8(a) is the measured PTE response when the receiver is implanted in a rat body located within the cavity resonator. As shown, the PTE response is shifted downwards resulting in a decrease in peak obtainable PTE. Indeed, an important consideration when using resonator cavity enabled WPT is how the presence of objects will perturb the system. The resonant frequency and $Q_{0}$ of a resonant cavity are very sensitive to the EM properties of its contents. It is for this reason resonant cavities are often used to measure the EM properties of materials [23].

In our application, the contents of the cavity will be a moving animal. At our operating frequencies, tissue will appear as a lossy dielectric [28]. Consequently, a downward shift in the resonant frequency and a drop in cavity $Q_{0}$ will occur. From our measurements, we observed a decrease in cavity $Q_{0}$ from 1038 to 480 . Also, we expect the coupling coefficient to be changing as the animal moves within the cavity. Additionally, the parameters $\left(Q_{0}\right.$ and $\left.L\right)$ of the receive resonator will also change; from our measurements we observed a decrease in receive resonator $Q_{0}$ (67.48 to 30.0 in animal) and a slight increase in coil $L$ (68 $\mathrm{nH}$ to $69 \mathrm{nH}$ in animal). We mitigate the change in receiver $Q_{0}$ by packaging the device within a capsule made of commercially available medical epoxy. This helps to isolate the device from animal tissue. All of these changes result in alterations to the circuit model, meaning that optimal PTE can still be achieved in implantation as long as measurements are made to account for the change in resonator $L, Q_{0}$, and $k_{12}$. 


\section{$>$ REPLACE THIS LINE WITH YOUR PAPER IDENTIFICATION NUMBER (DOUBLE-CLICK HERE TO EDIT) <}

\section{Custom Designed Implantable Device}

This section provides a description of our custom designed implantable device used for chronic in-vivo experiments within the cavity. The implantable device is our custom designed Bionode and includes our unique biaxial resonator and RF-toDC conversion circuitry module, all of which are encapsulated within a cylindrical package.

\section{A. Bionode System}

The Bionode is a custom designed fully implantable, fully wireless, and configurable bioelectric recording and stimulating device. Designed using only commercially available microelectronics, its features include two channels for biopotential acquisition, one channel for neuromodulation (stimulating), temperature sensing, electrode impedance measurement, and bi-directional telemetry. In this work, we utilized only the biopotential recording features and forward telemetry to extract ECG signals from freely behaving rats. On the Bionode, device tasks are directed by an nRF51822 (Nordic Semiconductor) system-on-chip (SoC) which includes an ARM ${ }^{\circledR}$ Cortex $^{\mathrm{TM}} \mathrm{M} 0$ microcontroller, 8/9/10-bit ADC, and a $2.4 \mathrm{GHz}$ transceiver. Wireless communication to and from a custom designed base station placed outside of the cavity resonator is used to stream data from the implanted device and to deliver directives such as desired sampling rate and stimulation parameters to the implanted device. The base station relays recorded data in real time to a custom software/graphical user interface (GUI) for illustration and storage. The power consumption of the device varies with the system's total sampling rate from $6.1 \mathrm{~mW}$ (total sampling rate $=5 \mathrm{kHz}$ ) to $13 \mathrm{~mW}$ (total sampling rate $=25 \mathrm{kHz}$ ).

\section{B. Biaxial Tunable Power Module}

Fig. 9 shows a schematic and photograph of the biaxial, tunable RF-to-DC conversion and voltage regulation circuitry. This system is referred to as the power module. The power module converts the RF energy, coupled from within the cavity, into a usable and stable DC supply for operation of the Bionode. Importantly, we design the power module using two receive coils oriented perpendicular to each other to achieve bidirectional coupling: a $7 \mathrm{~mm}$ diameter coil oriented in the $x$ axis and a $5 \mathrm{~mm}$ diameter coil oriented in the $y$-axis. Additionally, each coil is connected to its own J-inverter and rectifier circuit to enable independent resonator tuning. A third coil ( $z$-axis), J-inverter, and rectifier could easily be added to achieve truly omnidirectional powering. However, we omit a third axis to maintain miniaturization and simplicity of the total packaged system.

As shown in Fig. 9(a), the output of each J-inverter is connected to a voltage doubling rectifier circuit constructed from Schottky diodes and chip capacitors. A single SOT-363 bridge quad diode package (HSMS-282P) is used for both rectifiers for ease of fabrication. The rectified DC output voltage, $V_{\text {rect }}$, represents the combined DC voltage of both rectifiers and is connected to a $5.6 \mathrm{~V}$ Zener diode (Diodes Incorporated, BZT585B5V6T-7), which provides overvoltage protection to the voltage regulator (Analog Devices, ADP150ACBZ-1.8-R7CT). The voltage regulator provides a stable $1.8 \mathrm{~V}$ for the bioelectric recording device. This regulated

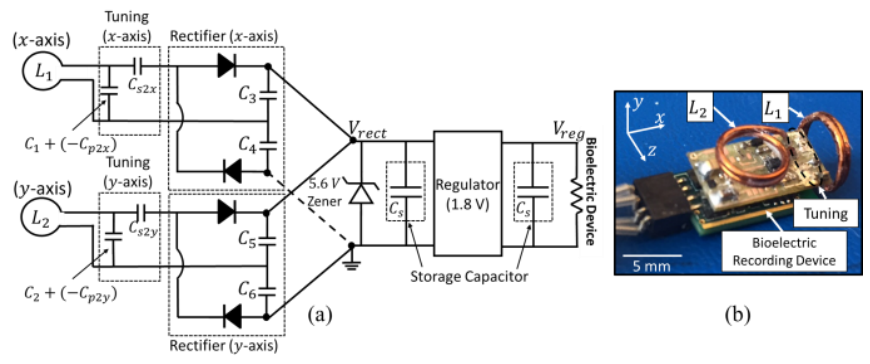

Fig. 9. Biaxial RF-to-DC conversion device. (a) Schematic diagram of biaxial power module. (b) Photograph of fabricated power module device PCB which is stacked on top of and electrically connected to the Bionode.

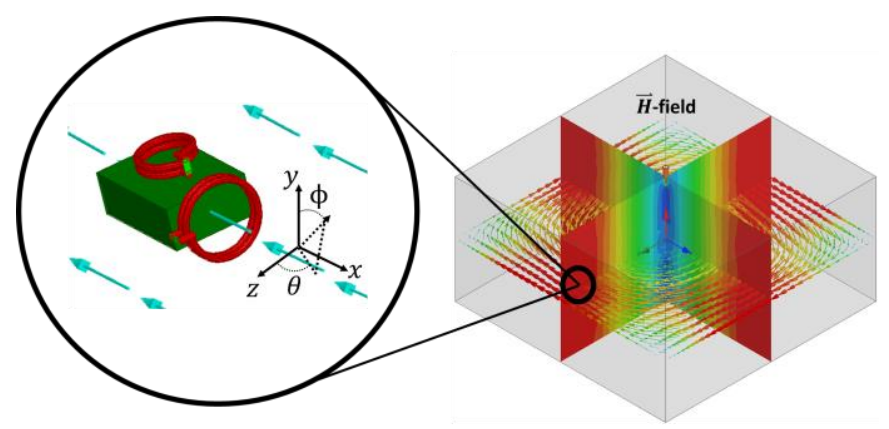

(a)

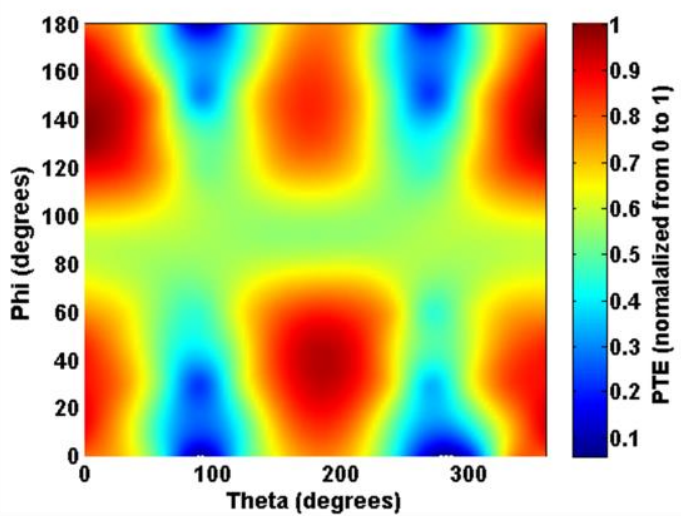

(b)

Fig. 10. Simulated power receive performance as a function of angular rotation of the biaxial arranged power receive system. (a) Orientation and coordinate reference. (b) Normalized (0 to 1) simulated PTE as a function of angular rotation (theta and phi).

supply is subsequently inverted to $-1.8 \mathrm{~V}$ to provide dual supplies for the Bionode's analog circuitry. Storage capacitors $(10 \mu \mathrm{F})$ on $V_{\text {rect }}$ and all regulator outputs buffer the power supply and minimize ripple on the DC supply lines.

\section{Biaxial PTE Performance}

Fig. 10 shows the normalized (from 0 to 1 ) simulated PTE performance of the biaxial coil system as a function of angular rotation (theta and phi). The data was acquired through an angular parametric sweep in HFSS. Indeed, only 4 angular conditions $\left(\mathrm{phi}=0^{\circ}\right.$, theta $=90^{\circ} ; \mathrm{phi}=180^{\circ}$, theta $=90^{\circ} ; \mathrm{phi}=$ $0^{\circ}$, theta $=270^{\circ} ; \mathrm{phi}=180^{\circ}$, theta $=270^{\circ}$ ) result in significant PTE degradation (normalized PTE < 0.3). Consequently, the biaxial system should be implanted in an advantageous 


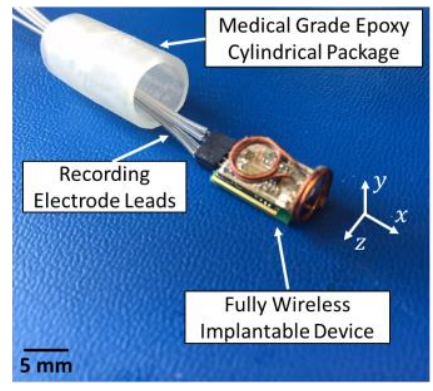

(a)

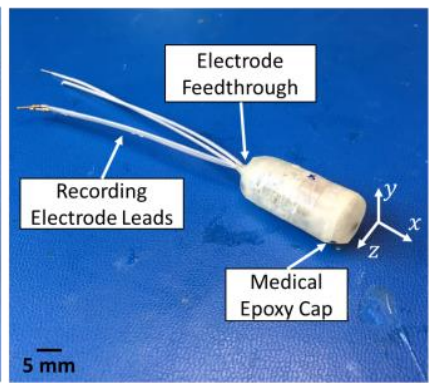

(b)
Fig. 11. (a) Pre-packaged and (b) fully packaged wireless implantable device with biaxial powering.

orientation to maximize orientation insensitivity when implanted in a freely behaving animal. A biaxial system implanted in the animal should have the $x$-axis, and associated coil [referenced to coordinate system in Fig. 10(a)], oriented along the length of the rodent body and the $y$-axis, and associated coil, oriented perpendicular to the rodent.

\section{Packaging}

Fig. 11 shows the pre-packaged and fully packaged system. We design the packages in a cylindrical form factor using biocompatible medical grade epoxy (Loctite ${ }^{\circledR}$ EA M-31CL Adhesive). The package has an inner diameter (ID) of $10.5 \mathrm{~mm}$ (to account for stacking of the Bionode and power module), an outer diameter (OD) of $14 \mathrm{~mm}$, and a length of $25 \mathrm{~mm}$. We place the implantable device within the cylindrical container and seal it with a medical epoxy lid. An electrode feedthrough is created at the end of the package and sealed with medical grade epoxy. The fully packaged device weighed $4.29 \mathrm{~g}$.

\section{CHRONIC IN-VIVO EXPERIMENT}

We demonstrate the functional utility of the cavity resonator WPT system through chronic in-vivo experiments involving freely behaving rats. A wireless powering fidelity metric is created to quantify continuous powering capability along with the wireless collection of ECG recordings from a freely behaving animal within the cavity resonator.

\section{A. Surgical Procedure}

Two rats (identified as R200 and ER34) weighing $197 \mathrm{~g}$ and $263 \mathrm{~g}$ were used in our in-vivo experiments. All procedures were approved by the Purdue Animal Care and Use Committee (PACUC) at Purdue University. Aseptic surgical technique was performed prior to and after each rat was anesthetized with isoflurane $\left(0.5 \%\right.$ - $3 \%$ in $\left.2 \mathrm{~L} / \mathrm{min} \mathrm{O}_{2}\right)$. A small incision was made on one side of the animal for device insertion and followed by tunneling to the lateral side for electrode placement (ECG Lead II configuration). To ensure adequate orientation insensitivity, we implanted and affixed the device such that the biaxial coils would be perpendicular to the $x$ and $y$ axes of the cavity [referenced to Fig. 7(a)] while the animal was in normal or standing position.

\section{B. In-vivo Experimental Set-up and Validation}

Fig. 12(a) shows the in-vivo experimental setup. A 335.0 $\mathrm{MHz}$ RF signal from a signal generator (Agilent EXG Vector Signal Generator N5172B), was fed into a power amplifier (PA,

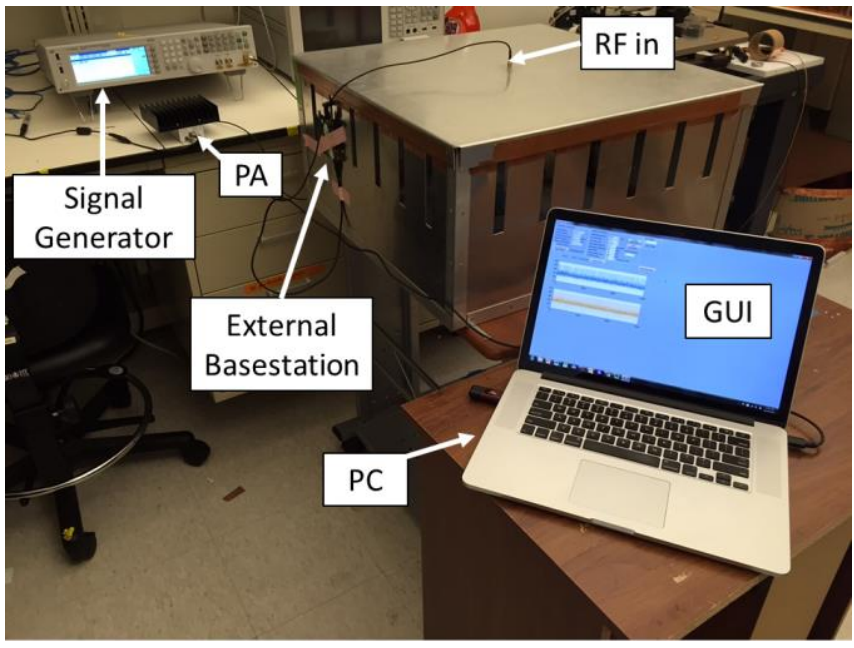

(a)

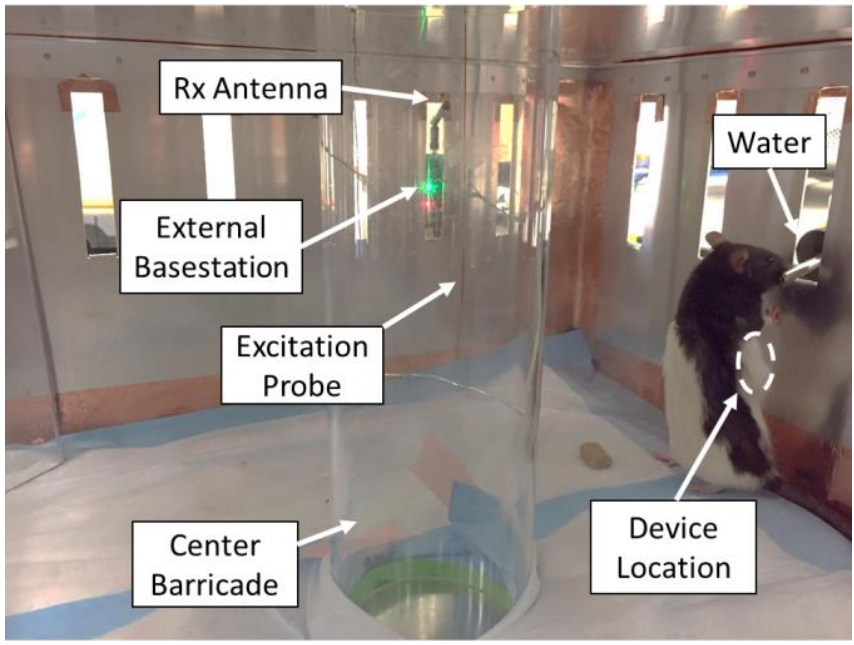

(b)

Fig. 12. (a) In-vivo experimental set-up and (b) interior view of cavity with rat within on post-op day 43. The green light on the external base station indicates device powering and capture of telemetered data.

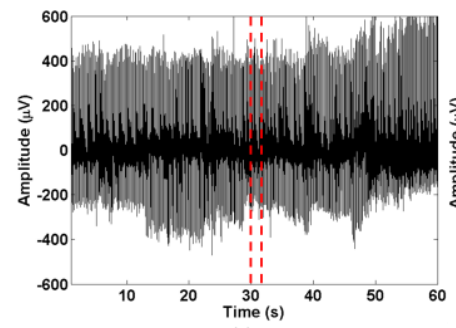

(a)

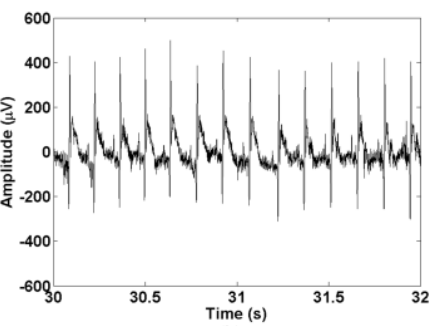

(b)
Fig. 13. Wirelessly acquired rat ECG signal from within the cavity (a) $60 \mathrm{~s}$ window and (b) 2 second segment.

Mini-Circuits ${ }^{\circledR}$ ZHL-1-2W) which connected directly to the excitation probe of the cavity. The high electric permittivity of biological tissues [28] act to decrease the resonant frequency of the cavity [see eqn. (1)]. Thus, to compensate for the presence of the animal, we excite the cavity at $335.0 \mathrm{MHz}$ instead of the calculated and measured frequency of $346.6 \mathrm{MHz}$. To maximize powering fidelity, all in-vivo experiments were conducted at $2 \mathrm{~W}$ input power into the cavity, the maximum rated power output of the PA. In order to robustly capture the 


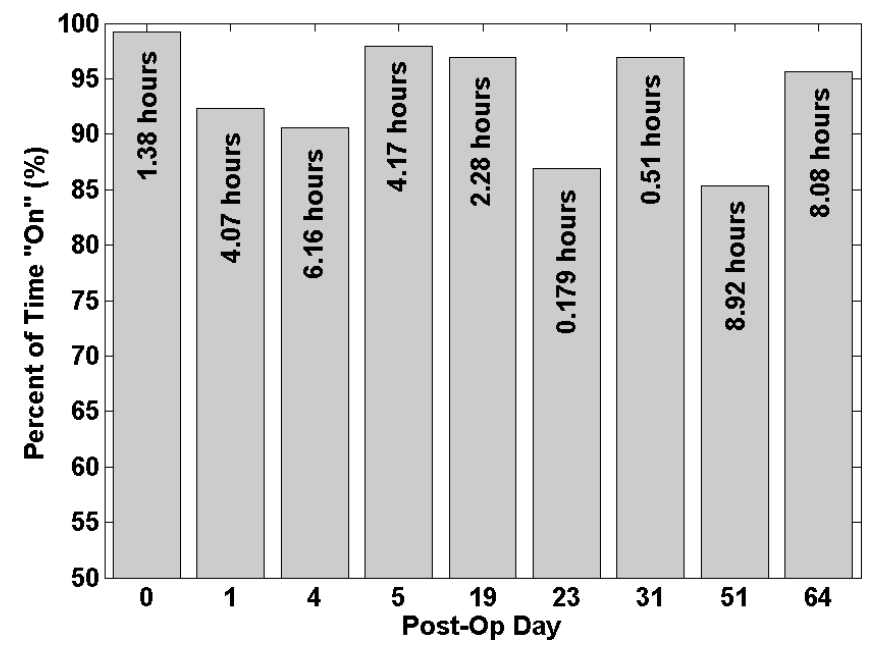

Fig. 14. Power fidelity distribution for rat R200 for nine separate recording sessions taken over a period of nine weeks. The recording session length is provided in each bar.

telemetered data, we positioned the receive antenna of the external base station directly into the cavity.

Fig. 12(b) shows an inside view of the cavity containing rat ER34 (post-operative day 43). Fig. 13 provides a snapshot of the acquired ECG signal (sampling rate of $2.5 \mathrm{kHz}$ ). Video clips of a freely behaving rat (standing and normal exploration) being wirelessly powered within the cavity can be found in Suppl. Video 1 and Suppl. Video 2.

\section{Evaluation of Powering Fidelity}

In order to evaluate the practical performance capability of the cavity resonator WPT system, we create a powering fidelity metric given by

$$
\% \text { On }=\frac{\text { no. of samples recieved }}{\text { no. of samples expected }} \times 100
$$

where no. of samples expected is determined by the length of the recording session multiplied by the sampling frequency. Since (24) is purely a powering fidelity metric, all received samples, including erroneous samples, were included in the calculation. Fig. 14 shows the powering fidelity for rat R200 over nine separate recording sessions of varying length up to post-operative day 64 . Over the course of the chronic study, the implanted device in rat ER34 had repositioned into a non-ideal orientation. Consequently, its powering fidelity was not included in Fig. 14. All recording sessions began directly after animal placement and closure of the cavity. This ensured that all powering fidelity measurements captured active, preacclimation, exploration behaviors which included standing, walking, and running. During long recording sessions $(>2$ hours), where acclimation to the cavity was achieved, animal activities were observed to also include resting behavior such as eating, drinking, grooming, and sleeping. As shown in Fig. 14 , we achieved a maximum powering fidelity of $99.24 \%$ for a 1.38 hour recording session. For the longer 8.92 hour and 8.08 hour recording sessions, we achieved a powering fidelity of $85.35 \%$ and $95.63 \%$, respectively. Power drop outs were observed to occur when the animal stood at angled positions

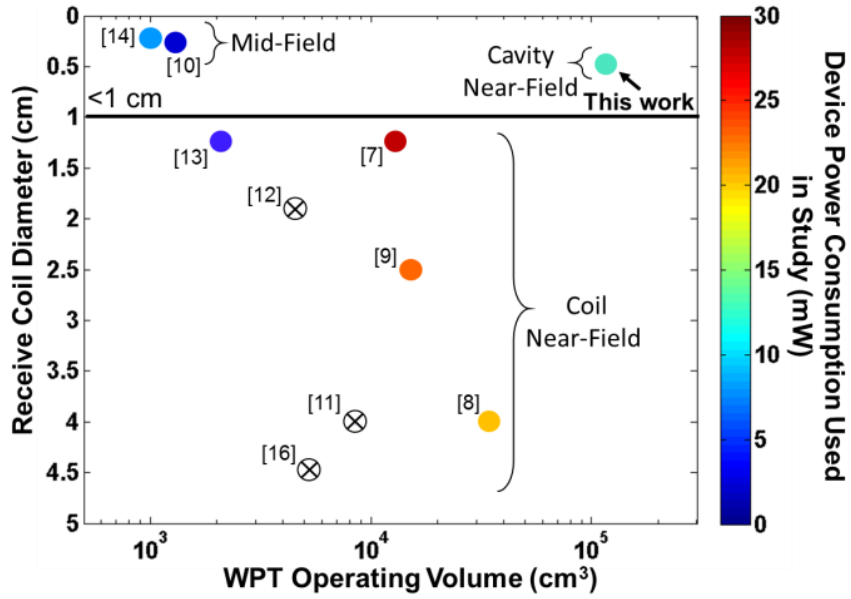

Fig. 15. Comparison of state-of-the-art WPT systems used for small animal studies. WPT systems approaching the upper right corner exhibit the largest operating volume with greatest device miniaturization capability. See Suppl. Table I for description of arena volume calculations for each reference and PTE performance comparison. Circles with an " $X$ " represent references which did not describe or demonstrate wireless powering of a medical device.

against the side walls and center barrier where the biaxial system became ineffective at capturing the $\vec{H}$-field of the $\mathrm{TM}_{110}$ resonant mode. Overall, across 9 recording sessions, with observed natural exploration and resting behaviors, we achieved an average powering fidelity of $93.53 \%$. Indeed, the biaxial and optimally tuned receive resonator system is sufficient for nearly continuous device operation.

\section{Specific Absorption Rate Considerations}

Regulatory agencies such as the FCC measure tissue exposure to RF energy by the specific absorption rate (SAR). Although multiple standards exist, the limits imposed to prevent damage to human tissue by IEEE is $10 \mathrm{~W} / \mathrm{kg}(20 \mathrm{~W} / \mathrm{kg}$ in extremities) averaged over $10 \mathrm{~g}$ of tissue [29]. Although nonhuman animals do not have such standards, any freely behaving animal environment should not include RF exposure that results in behavioral changes of the animal. Moreover, staying below SAR limits will ensure safety for the user and promote use across the scientific community.

In this work, the cavity is fed $2 \mathrm{~W}$ at $335.0 \mathrm{MHz}$. This is well below the power and frequency levels associated with similar work which showed safe SAR exposure as high as $4 \mathrm{~W}$ at 1.5 $\mathrm{GHz}$ [19]. As we are operating with a similar structure, lower frequency, lower power, and have observed no behavioral changes in any of our animal experiments, we predict our animal subjects remain well below $10 \mathrm{~W} / \mathrm{kg}$.

\section{CONCLUSION}

In this paper, we have developed and demonstrated a cavity resonator based WPT system for long-term studies involving freely behaving rats. Strategic cavity design and construction techniques were presented which promoted its utility as a large and engaging animal housing environment while simultaneously maintaining EM performance for WPT. Additionally, we utilized our unique circuit model and simple optimal IM methodology for maximizing WPT performance. A miniaturized and tunable biaxial receive resonator power 
module system, formed of $7 \mathrm{~mm}$ and $5 \mathrm{~mm}$ diameter coils, was designed to improve device orientation insensitivity. As a result, we achieved sufficient WPT performance to operate a sophisticated bioelectric recording device, in-vivo, with an average powering fidelity of $93.54 \%$ to a freely behaving animal within the cavity. Finally, we conclude with Fig. 15 which provides a comparison of our work with current state-ofthe-art systems. Indeed, this work enables wireless powering of devices formed with nearly the smallest receive coil diameters implanted in rodents freely moving within the largest volume.

\section{FUTURE DIRECTIONS}

Demonstrated previously in [22], cavity resonator WPT systems are capable of delivering power to multiple devices simultaneously; future cavity systems could be optimized to power devices implanted in multiple animals within a single large cavity resonator. A dynamic tuning solution for cavity probe length and on-board capacitors would be ideal to account for the moving animals within the cavity to help achieve similar or superior performance.

\section{ACKNOWLEDGMENT}

This work was sponsored by the Defense Advanced Research Projects Agency (DARPA) BTO under the auspices of Dr. Doug Weber through the Space and Naval Warfare Systems Center, Grant/Contract No. N6601-14-24056. The authors thank D. Pederson and Z. Wang for contribution in software development, G. Albors for assistance in device fabrication, D. McMillan for aiding in development and construction of the cavity resonators, and C. Quinkert for assistance in PCB design.

\section{REFERENCES}

[1] S. B. Lee et al., "An Inductively Powered Scalable 32-Channel Wireless Neural Recording System-on-a-Chip for Neuroscience Applications," IEEE Transactions on Biomedical Circuits and Systems, vol. 4, no. 6, pp. 360-371, Dec, 2010.

[2] S. T. Lee et al., "A Miniature, Fiber-Coupled, Wireless, Deep-Brain Optogenetic Stimulator," IEEE Transactions on Neural Systems and Rehabilitation Engineering, vol. 23, no. 4, pp. 655-664, Jul, 2015.

[3] Q. Xu et al., "A Fully Implantable Stimulator With Wireless Power and Data Transmission for Experimental Investigation of Epidural Spinal Cord Stimulation," IEEE Transactions on Neural Systems and Rehabilitation Engineering, vol. 23, no. 4, pp. 683-692, Jul, 2015.

[4] H. Bhamra et al., "A $24 \mu \mathrm{W}$, Batteryless, Crystal-free, Multinode Synchronized SoC "Bionode" for Wireless Prosthesis Control," IEEE Journal of Solid-State Circuits, vol. 50, pp. 2714-2727, Nov. 2015.

[5] T. A. Szuts et al., "A wireless multi-channel neural amplifier for freely moving animals," Nature Neuroscience, vol. 14, no. 2, pp. 263-U363, Feb, 2011.

[6] D. Fan et al., "A Wireless Multi-Channel Recording System for Freely Behaving Mice and Rats," Plos One, vol. 6, no. 7, Jul 12, 2011.

[7] C.-W. Chang, and J.-C. Chiou, "A Wireless and Batteryless Microsystem with Implantable Grid Electrode/3-Dimensional Probe Array for ECoG and Extracellular Neural Recording in Rats," Sensors, vol. 13, no. 4, pp. 4624-4639, Apr, 2013.

[8] U.-M. Jow et al., "EnerCage: A Smart Experimental Arena With Scalable Architecture for Behavioral Experiments," IEEE Transactions on Biomedical Engineering, vol. 61, no. 1, pp. 139-148, Jan, 2014.

[9] B. Lee et al., "A Smart Wirelessly Powered Homecage for Long-Term High-Throughput Behavioral Experiments," IEEE Sensors Journal, vol. 15, no. 9, pp. 4905-4916, Sep, 2015.
[10] A. J. Yeh et al., "Wirelessly powering miniature implants for optogenetic stimulation," Applied Physics Letters, vol. 103, no. 16, Oct 14, 2013.

[11] N. Soltani et al., "Cellular Inductive Powering System for Weakly-Linked Resonant Rodent Implants," Biomedical Circuits and Systems Conference. pp. 350-353, 2013.

[12] K. Eom et al., "A wireless power transmission system for implantable devices in freely moving rodents," Medical \& Biological Engineering \& Computing, vol. 52, no. 8, pp. 639-651, Aug, 2014.

[13] E. G. Kilinc et al., "A System for Wireless Power Transfer of MicroSystems In-Vivo Implantable in Freely Moving Animals," IEEE Sensors Journal, vol. 14, no. 2, pp. 522-531, Feb, 2014.

[14] K. L. Montgomery et al., "Wirelessly powered, fully internal optogenetics for brain, spinal and peripheral circuits in mice," Nat Meth, vol. advance online publication, 08/17/online, 2015.

[15] J. S. Ho et al., "Wireless power transfer to deep-tissue microimplants," Proceedings of the National Academy of Sciences of the United States of America, vol. 111, no. 22, pp. 7974-7979, Jun, 2014.

[16] S. A. Mirbozorgi et al., "A Smart Multicoil Inductively Coupled Array for Wireless Power Transmission," IEEE Transactions on Industrial Electronics, vol. 61, no. 11, pp. 6061-6070, Nov, 2014.

[17] U.-M. Jow, and M. Ghovanloo, "Modeling and Optimization of Printed Spiral Coils in Air, Saline, and Muscle Tissue Environments," IEEE Transactions on Biomedical Circuits and Systems, vol. 3, no. 5, pp. 339347, Oct, 2009.

[18] U. M. Jow, and M. Ghovanloo, "Geometrical Design of a Scalable Overlapping Planar Spiral Coil Array to Generate a Homogeneous Magnetic Field," IEEE Transactions on Magnetics, vol. 49, no. 6, pp. 2933-2945, Jun, 2013.

[19] J. S. Ho et al., "Self-Tracking Energy Transfer for Neural Stimulation in Untethered Mice," Physical Review Applied, vol. 4, no. 2, Aug, 2015.

[20] H. Mei et al., "Optimal Wireless Power Transfer to Systems in an Enclosed Resonant Cavity," IEEE Antennas and Wireless Propagation Letters, vol. 15, pp. 1036-1039, 2015.

[21] M. J. Chabalko, and A. P. Sample, "Resonant cavity mode enabled wireless power transfer," Applied Physics Letters, vol. 105, no. 24, Dec, 2014.

[22] M. J. Chabalko, and A. P. Sample, "Three-Dimensional Charging via Multimode Resonant Cavity Enabled Wireless Power Transfer," IEEE Transactions on Power Electronics, vol. 30, no. 11, pp. 6163-6173, Nov, 2015.

[23] D. M. Pozar, Microwave Engineering, 4 ed.: John Wiley \& Sons, Inc., 2012.

[24] R. A. Bercich et al., "Far-Field RF Powering of Implantable Devices: Safety Considerations," IEEE Transactions on Biomedical Engineering, vol. 60, no. 8, pp. 2107-2112, Aug, 2013.

[25] P. Couffignal et al., "Equivalent-circuit of a cavity coupled to a feeding line and its dependence on the electric or magnetic nature of output coupling structure," Iee Proceedings-H Microwaves Antennas and Propagation, vol. 139, no. 3, pp. 221-226, Jun, 1992.

[26] J.-S. Hong, Microstrip Filters for RF/Microwave Applications, 2nd ed.: John Wiley \& Sons, Inc., 2011.

[27] D. Kajfez, and E. J. Hwan, "Q-factor mesurement with network analyzer," IEEE Transactions on Microwave Theory and Techniques, vol. 32, no. 7, pp. 666-670, 1984.

[28] S. Gabriel et al., "The dielectric properties of biological tissues .2. Measurements in the frequency range $10 \mathrm{~Hz}$ to $20 \mathrm{GHz}$," Physics in Medicine and Biology, vol. 41, no. 11, pp. 2251-2269, Nov, 1996.

[29] IEEE Standard C95.1, "IEEE Standard for Safety Levels with Respect to Human Exposure to Radio Frequency Electromagnetic Fields, $3 \mathrm{kHz}$ to $300 \mathrm{GHz}, " 2005$. 